Historia Contemporánea 58, 2018: 625-642

ISSN: $1130-2402-$ e-ISSN: 2340-0277

DOI: $10.1387 /$ hc. 20113

\title{
30 AÑOS DE LA REVISTA HISTORIA CONTEMPORÁNEA
}

\section{YEARS OF THE JOURNAL «HISTORIA CONTEMPORÁNEA»}

\author{
Mariano Esteban de Vega \\ Universidad de Salamanca
}

ORCID: https://orcid.org/0000-0002-0318-1455

Resumen: La revista Historia Contemporánea fue creada en 1988, dentro del departamento de Historia Contemporánea de la Universidad del País Vasco, por iniciativa de Manuel Tuñón de Lara, uno de los más influyentes historiadores españoles de su época. Desde entonces, ha sido una de las revistas españolas de historia más prestigiosas y, en los últimos años, también una de las que han alcanzado un mayor impacto editorial.

Este artículo realiza una historia de los treinta años de esta publicación e identifica sus fases principales. Su evolución se inserta además en los cambios experimentados por la historiografía española de su tiempo, de la que ha sido uno de sus principales referentes. Tanto sus contenidos como su configuración actual resultan significativos de la progresiva asimilación por el medio académico español de las pautas seguidas a escala internacional por las publicaciones científicas.

Palabras Clave: Historia de la historiografía; historiografía española; revistas de historia; Manuel Tuñón de Lara.

Abstract: The journal «Historia Contemporánea» was founded in 1988, within the Department of Contemporary History at the University of País Vasco. It was initiated by Manuel Tuñón de Lara, one of the most relevant Spanish historiographers at the end of Francoism. Since then, it has been one of the most prestigious Spanish history journals and, in recent years, it has also been one of the journals with the most editorial impact.

This article includes this publication's thirty years of history, and identifies its initial phases. Its evolution goes hand in hand with the changes that its co- 
etaneous Spanish historiography has experienced, of which it has been a fundamental role model. Both its content and its current layout are significant with regards to the progressive assimilation of the internationally followed patterns in scientific journals by the Spanish academic media.

Keywords: Historiography history; Spanish historiography; history journals; Manuel Tuñón de Lara. 
En 1988, hace ahora treinta años, vio la luz el número 1 de la revista Historia Contemporánea. Vinculada directamente al departamento de Historia Contemporánea de la Universidad del País Vasco, y bajo la dirección de Manuel Tuñón de Lara, uno de los grandes referentes de la historiografía española de las décadas de los sesenta, setenta y ochenta (las del final del franquismo, la transición y la primera democracia), la revista constituyó una de las numerosas iniciativas editoriales que en aquellos años de expansión económica y consolidación académica protagonizaron los departamentos y facultades de Historia de las universidades españolas $^{1}$. Miquel Marín Gelabert se ha referido en varias ocasiones a aquella coyuntura particular de efervescencia asociativa, promoción de congresos y dinamismo editorial, que significó un verdadero «cambio de guardia» en el contemporaneísmo español, una refundación generacional y profesional que trataba de dejar atrás la herencia de la historiografía anterior ${ }^{2}$. Unos años marcados también por una cierta perplejidad, pues el paradigma al fin triunfante en España de una historia científica en diálogo con las ciencias sociales tuvo que enfrentarse $-\mathrm{y}$ lo hizo con desconcierto - a los primeros ecos de la crisis universal de los grandes modelos y a la irrupción de las propuestas postmodernas, a una multiplicación imparable de las formas de hacer historia y a una progresiva hiperespecialización temática y metodológica, con sus correspondientes peligros de fragmentación ${ }^{3}$.

Desde entonces, Historia Contemporánea no solo ha cubierto una prolongada trayectoria, sino que ha sido una de las revistas españolas más prestigiosas y - en los últimos años - una de las que pueden mostrar da-

1 Antes de la publicación de Historia Contemporánea cabe reseñar la aparición, en el ámbito editorial universitario, de las revistas Estudis d'Història Contemporània del País Valencià, en la Universidad de Valencia (1979); Investigaciones Históricas. Épocas Moderna y Contemporánea, en la Universidad de Valladolid (1979); Cuadernos de Historia Moderna y Contemporánea, en la Universidad Complutense (1980), que en 1988 dio lugar a Cuadernos de Historia Contemporánea; Anuario de Historia Contemporánea, en la Universidad de Granada (1981); Anales de Historia Contemporánea, en la Universidad de Murcia (1982); Revista de Historia Contemporánea, en la Universidad de Sevilla (1982); Studia Historica. Historia Contemporánea, en la Universidad de Salamanca (1983) y, un poco más tarde, el mismo año que Historia Contemporánea, de la revista Historia Social, vinculada al centro asociado de la UNED de Alzira-Valencia (1988). La revista Ayer, dependiente de la Asociación de Historia Contemporánea y llamada a convertirse también, como Historia Contemporánea, en publicación de referencia del contemporaneísmo español, nació en 1991.

2 Marín Gelabert, 2001 y 2013.

3 Esteban de Vega, 2009, pp. 9-15. 
tos de impacto editorial más concluyentes, marcando el camino de un proceso de renovación que otras muchas publicaciones universitarias españolas no han conseguido aún acometer. Estas páginas constituyen un breve relato y un primer análisis de este recorrido de tres décadas, inserto en la evolución general que la historiografía española contemporánea ha seguido en este tiempo. Una época en la que, con todos los matices que puedan plantearse, esta experimentó un avance muy notable, alcanzando un nivel de producción en cantidad y calidad que, para Pérez Garzón, permitiría afirmar incluso que el retraso derivado del aislamiento y la represión intelectual del franquismo había quedado superado ${ }^{4}$.

\section{La primera época: la dirección de Manuel Tuñón de Lara, 1988-1996}

Como resulta habitual, la primera entrega de Historia Contemporánea llevó incorporada una «Presentación», en la que se manifestaban los objetivos de la revista. Firmada por su director, Manuel Tuñón de Lara, se encuentran en ella muchas de las que fueron las principales constantes de su labor como historiador, formado vocacionalmente en el exilio bajo la influencia de las principales corrientes de la historia social vigentes en la Francia de la postguerra, intérprete de una versión singular del materialismo histórico y, sobre todo, agente sustancial de la renovación y apertura de la historiografía española de su tiempo ${ }^{5}$.

En primer lugar, se anuncia en ella un deseo de trascender el ámbito meramente universitario: sus promotores eran «historiadores de profesión y de vocación [...] convencidos de que la Universidad se proyecta sobre la sociedad entera por medio de la creación y difusión de cultura y de la formación profesional»; del mismo modo, aunque la revista se definía como «eminentemente profesional [...], dirigida en primer lugar a todos los historiadores que trabajan en la investigación y/o en la docencia, tanto en universidades y centros de análogo nivel, como en los grados medio y básico de la enseñanza», también aspiraba a interesar a «todos los estudiantes universitarios de historia». A continuación, se lleva a cabo una declaración explícita de adhesión a la libertad intelectual: conceptos básicos de la tarea del historiador, previos y condicionantes de

\footnotetext{
4 Pérez Garzón, 2009.

5 Entre otros ejemplos posibles, Granja y Reig Tapia (eds.), 1993, y Granja, Reig Tapia y Miralles (eds.), 1999.
} 
todos los demás, serían el «pluralismo metodológico, libre debate científico, la independencia de nuestro trabajo con respecto a cualquier corriente organizada de opinión y, en suma, la tolerancia en el terreno de la investigación científica y de su expresión oral y escrita». Además, se presenta una comprensión de la historia que abraza nociones muy diversas («memoria colectiva sistematizada», «ciencia de los hombres en el tiempo», «explicación de un proceso de cambios sociales y de resistencias inmovilistas a los mismos»), pero cuya utilidad sería indudable, pues «sin conocer el pasado con la mayor precisión y riqueza posible, mal puede comprenderse el presente y son nulas nuestras armas prospectivas». Por lo demás, la revista realiza un llamamiento a la colaboración con las ciencias sociales inequívocamente enraizado en la renovación historiográfica iniciada en la Europa de entreguerras, en clave de historia social, y consolidada, hasta convertirse en hegemónica, en las décadas que siguieron a la Segunda Guerra Mundial:

Son también principios que hacemos nuestros los de interdisciplinariedad y de cooperación con todas las ramas afines del saber, postulando en este sentido una constante operación simbiótica; así con la economía, la sociología, la ciencia política, la antropología, la filología, la estadística, la arqueología y otras muchas que omitimos. Pensamos que la historia debiera realizar una vasta operación de síntesis de todas las ciencias en el pasado y que, por consiguiente, necesita también de un múltiple enriquecimiento metodológico al contacto con las disciplinas citadas.

En esa misma línea, aparece la aspiración, muy de la época, una vez más de inspiración muy francesa, a una historia en la que los distintos sectores o especialidades fueran «capaces de articularse e integrarse en una concepción global del pasado»; en definitiva, «la historia a secas, que preferimos llamar global, sin duda de difícil realización, pero a la que no renunciamos como meta» ${ }^{6}$.

Por fin, atendiendo al contexto de despliegue que entonces se estaba produciendo del Estado de las Autonomías, la revista reservaba también, programáticamente, una atención especial a su entorno vasco:

${ }^{6}$ De hecho, para Julio Aróstegui una de las ideas centrales en la obra historiográfica de Tuñón de Lara sería, precisamente, la de la interdisciplinariedad como vía para lograr una historia global o total; Aróstegui, 1993, p. 168. 
Nos guía el talante de universalidad y de respeto al primado de la razón que va implícito en la esencia misma de la Universidad. Abiertos a todos los horizontes y a todos los intercambios, no olvidamos, naturalmente, el área importante que en nuestra empresa corresponde a la historia del País Vasco en todos sus aspectos.

Para desarrollar estos presupuestos, la revista estaría estructurada en cuatro grandes apartados: uno dedicado a un tema monográfico, otro apartado misceláneo (para recoger «investigaciones sobre temas o asuntos concretos, así como las reflexiones, debates, estados de la cuestión, etc.»), una tercera sección sobre «documentación» (que apenas llegó a desarrollarse) y, finalmente, una cuarta parte dedicada a recensiones, notas de libros e informaciones bibliográficas o sobre congresos y coloquios.

Historia Contemporánea siguió estas pautas marcadas por Manuel Tuñón de Lara durante sus primeros nueve años de vida (desde su fundación en 1988 hasta la publicación del número 15, en el segundo semestre de 1996). La revista no consiguió en ese periodo una periodicidad estable: fue anual en los dos primeros años (1988 y 1989), bianual en los cuatro siguientes (de 1990 a 1993), anual de nuevo en 1994 y 1995 y bianual otra vez en 1996 (año en el que además se publicó un número doble, el 13$14)^{7}$. Por otra parte, como era común entonces en las revistas españolas, a veces alivió sus problemas de periodicidad acogiendo las actas de algún congreso: así, el número 4 (1990) se dedicó íntegramente al congreso «Cambios sociales y modernización», celebrado en Vitoria en ese mismo año; y el dosier del número 7 (1992), «Historiografía contemporánea reciente», incorporó las conferencias de un curso de verano organizado el año anterior por la Universidad del País Vasco. Como efecto secundario de estos problemas de la relativa irregularidad en la publicación de las sucesivas entregas, los volúmenes alcanzaron a menudo una extensión algo excesiva, generalmente muy por encima de las 300 páginas, superando en algunas ocasiones las 400 y, en el número doble 13-14, las 500.

7 En alguna ocasión, el director aludió con cierto humor a esta relativa irregularidad. Así, en la presentación del número 8 indicaba: «Una vez más la batalla contra el reloj y la carrera de obstáculos que representa la conjunción del calendario académico y otros como el laboral, el gregoriano, etc., con su tejido no ya de puentes sino de auténticos "viaductos", nos obliga a aparecer con un breve retraso de semanas. Esperamos que la benevolencia de los lectores sepa disculparnos». También en el número 10 se reconoce el retraso en la salida del volumen, originada por «la proximidad de los exámenes, de la preparación de cursos de verano y otras actividades académicas». 
La estrecha vinculación de la revista con la figura de su fundador resulta manifiesta en el hecho de que los primeros números de Historia Contemporánea incorporasen siempre introducciones firmadas por el propio Tuñón de Lara, en las que el director presentaba los contenidos del conjunto del volumen. Pero quizá la prueba más relevante de que estamos ante lo que Ricardo Miralles consideró «una empresa historiográfica con autor», el «último legado de Manuel Tuñón de Lara a la historiografía española contemporánea», fuera la naturaleza de los temas elegidos para los dosieres de la revista, muy representativos de la visión de la historia de Tuñón de Lara y de sus principales preocupaciones como historiador. En palabras del propio Miralles:

La colección de los quince números de Historia Contemporánea dirigidos por Tuñón de Lara abarca un conjunto de cuatro grandes apartados que incluyen Teoría, método e historiografía, Historia Social, Historia de las ideologías y de las mentalidades e Historia Política. Cualquier conocedor de su obra podrá estar de acuerdo en que las principales preocupaciones historiográficas de Tuñón están presentes en estos cuatro apartados. Tan sólo quedó sin abordar un quinto y último objeto historiográfico que él cultivó con especial aprecio, la Historia de la cultura, sobre la que tenía pensado reflexionar con motivo del centenario del $98^{8}$.

En efecto, temáticamente la revista mostró entonces una clara predilección por determinadas épocas de la historia de España: ante todo, la II República (objeto de los dosieres de los números 1,6 y 11 y, parcialmente, del 10), la Restauración (números 3 y 10) y, en una ocasión, la «revolución burguesa» a caballo entre los siglos XVIII y XIX (número 2). La reflexión historiográfica, el interés por la fundamentación teórica de la disciplina y por la discusión de las herramientas utilizadas por las diversas subdisciplinas, estuvieron además profusamente representadas en esta primera etapa de Historia Contemporánea, siendo objeto de la parte monográfica de los números 5,7 y 9 y de una buena cantidad de artículos repartidos por la mayoría de los números ${ }^{9}$. La perspectiva dominante en los estudios publicados entonces en la revista fue,

8 Miralles, 1997, p. 14.

9 En la presentación del número 3 Tuñón de Lara indicó incluso que «abrimos una sección de problemas historiográficos con el detenido estudio que nuestro colega Julio Aróstegui (catedrático de la Universidad Complutense y miembro del consejo asesor de Historia Contemporánea) ha elaborado sobre un tema tan polémico e interesante como es la última reedición ampliada del profesor Bollotten sobre la guerra civil española». 
desde luego, la de la historia social: al analizar la transición del Antiguo Régimen a la sociedad burguesa en el número 2; al conmemorar el centenario del primero de mayo en el número 3; y, naturalmente, al abordar el estudio de la relación entre cambios sociales y modernización en el 4, la Historia social y las mentalidades en el 5, la historiografía contemporánea reciente en el 7, el estudio de las elites en el 8 e incluso la «nueva historia política», en estrecha relación con la historia social, en el 9.

Por lo demás, la revista mostró en esta época un excelente nivel académico. Entre los colaboradores figuraron, desde luego, muchos de los profesores del departamento de Historia Contemporánea de la Universidad del País Vasco, que componían el Consejo de Redacción ${ }^{10}$. Pero a partir del número 2 la revista contó también con un prestigioso Consejo Asesor, formado por destacados hispanistas franceses (Jean-François Botrel, Carlos Serrano y Jacques Maurice), estadounidenses (Gabriel Jackson y Edward Malefakis), alemanes (Walther Bernecker) y británicos (Christopher H. Cobb, Frances Lannon y Paul Preston), junto a cinco de los más relevantes historiadores españoles (Julio Aróstegui, Miguel Artola, Albert Balcells, Juan José Carreras y Juan Pablo Fusi). Ello facilitó sin duda que una parte importante de los artículos que vieron la luz en la revista fueran firmados por historiadores ajenos a la Universidad del País Vasco, tanto de otras universidades españoles como extranjeras.

A partir del número 11, publicado en 1994, la presencia de Manuel Tuñón de Lara en la revista empezó a diluirse. Sus presentaciones fueron haciéndose mucho más breves (números 11 y 12) hasta desaparecer desde el número 13-14. Por otro lado, cada número comenzó ya a tener un coordinador, salido del Consejo Asesor o del Consejo de Redacción - Julio Aróstegui el 11, dedicado a «La militarización de la política durante la II República»; Javier Fernández Sebastián y José María Portillo el 12, sobre «Historia y Derecho»; Joseba Agirreazkuenaga el 13-14, titulado «A vueltas con el sujeto»; Ricardo Miralles el 15, «Nombres propios para una diplomacia»-, y dicho coordinador pasó a encargarse de realizar la correspondiente introducción ${ }^{11}$. En la presentación del volumen 13-14, pu-

10 De hecho, en esta época se cumplió casi siempre con el propósito fundacional de informar sobre publicaciones relacionadas con la historia del País Vasco, incluso - a partir del número 5- sobre tesis leídas con esa temática. Este apartado desapareció definitivamente de la revista, sin embargo, a partir del número 13-14.

11 Con la excepción del número 12, en el que la presentación del volumen corrió a cargo no de los coordinadores sino del profesor Francisco Tomás y Valiente. 
blicado en 1996, Joseba Agirreazkuenaga, director del número y cosecretario de la revista, dejaba traslucir que se acercaba el final de una época, haciendo ya un breve balance de los doce primeros volúmenes, en el que destacaba que, sobre todo, Historia Contemporánea había conseguido «definir una trayectoria temática y temporal»: por un lado, «una permanente preocupación por los grandes temas, es decir, aquellos que implican o añaden un halo de globalidad»; por otro, una atención preferente a «los problemas referidos al Estado español (institucionalización, política, historia social), particularmente los del tiempo de la II República». Finalmente, se apuntaba una aspiración que posteriormente resultaría medular para la revista, la búsqueda de la internacionalización:

Uno de los problemas historiográficos de la actualidad reside en «saber ubicarse» para encontrar una vía de investigación, comunicación y de práctica historiográfica conectada con problemas de la historiografía internacional. Un objetivo que debemos compartir los investigadores de la comunidad universitaria y científica. Precisamente, esta revista y sus monográficos, aspiran a constituirse en una eficaz red de comunicación e intercambio.

Ese mismo año 1996, el número 15 publicó una «Presentación», firmada por el otro secretario de la revista, Ricardo Miralles, coordinador también de ese volumen, que incluía una despedida de Manuel Tuñón de Lara, quien por su deteriorada salud ya no podía seguir ocupándose de la dirección. De hecho, Manuel Tuñón de Lara falleció el 25 de enero de 1997, noticia de la que informó el Consejo de Redacción en el número 16, publicado en 1997, consignando «unas palabras de homenaje y despedida a quien ha sido artífice indiscutible de esta revista de Historia Contemporánea, y maestro de historiadores».

\section{La transformación de la revista, 1997-2017}

Manuel Tuñón de Lara fue sucedido en la dirección de Historia Contemporánea por Manuel González Portilla, catedrático en la Facultad de Ciencias Sociales y de la Comunicación de la Universidad del País Vasco $\mathrm{y}$, desde la fundación de la revista, vicedirector de esta. González Portilla ejerció como director durante veintiún años, al término de los cuales se había producido una profunda transformación de la publicación, crecientemente homologable a las grandes revistas internacionales de historia. 
Los primeros años de la dirección de González Portilla marcaron una progresiva consolidación de la revista. En 1997 y 1998 siguió teniendo periodicidad anual, pero a partir de 1999 alcanzó una regularidad definitiva como revista semestral, con la publicación de dos números por año. Al principio, ello fue posible a veces, como en la etapa anterior, incorporando las actas de algunos congresos organizados por el departamento de Historia Contemporánea de la Universidad del País Vasco o por el Instituto de Historia Social «Valentín de Foronda» de Vitoria. Así sucedió en el número 18 (1999), titulado «Mortalidad infantil y condiciones de vida en la Europa del sur», que recogió en su parte monográfica las ponencias y algunas comunicaciones presentadas a la sesión «La transición de la mortalidad infantil y juvenil» del IV Congreso de Demografía Histórica (Bilbao, 1995); y también en el 19 (1999), donde se publicaron algunas de las ponencias y comunicaciones del Congreso «Cuba y España. Pasado y Presente de una Historia Común» (Vitoria, 1998). Pero esta práctica resultó ocasional y, como veremos después, acabó desapareciendo.

La estructura de la revista también varió ligeramente, eliminándose de modo definitivo la información sobre bibliografía vasca y definiendo con mayor claridad, aparte de las secciones fundamentales, monográfica y miscelánea, la dedicada a reseñas y comentarios de libros y la que recogía informaciones sobre congresos.

Por otra parte, como había sucedido ya en los últimos números de la etapa de la dirección de Tuñón de Lara, la revista dejó de incluir una presentación del director y cada volumen incorporó presentaciones de los coordinadores del dosier: en el número 16, dedicado a «Marginación, desigualdad y poder», de Juan Gracia Cárcamo y Lola Valverde; en el 17, titulado «El Estado en España», de Antonio Morales Moya y María Dolores de la Calle Velasco, profesores de la Universidad de Salamanca; en el 19, de Félix Luengo Teixidor y Pilar Pérez-Fuentes; en el 20, dedicado a «El hispanismo y la historia contemporánea de España», de Ricardo Miralles; en el 21, titulado «Estudios de género», de José Javier Díaz Freire.

La revista perdió así la impronta personal, muy ligada a la figura de su director, que había tenido en su primera etapa. Del mismo modo, los temas elegidos para los distintos dosieres fueron más diversos: algunos, como es lógico, encajaban plenamente en las preocupaciones tuñonianas (por ejemplo, el dedicado al hispanismo), pero otros implicaban una apertura temática (como los consagrados a la marginación y la pobreza o a los estudios de género) o una reflexión sobre aspectos teóricos y metodológicos profundamente renovada. 
La consolidación y regularización de la revista fue acompañándose de algunas modificaciones formales. A partir del número 20, en el año 2000, los volúmenes correspondientes a un mismo año se publicaron con paginación consecutiva, formando un conjunto. El número 21, ese mismo año, apareció precedido de un texto titulado «A los lectores» en el que se indicaba que Historia Contemporánea iniciaba una nueva etapa, encaminada a la «consolidación de su proyecto como portavoz cualificado de la producción historiográfica contemporánea tanto del País Vasco como de España». Para ello, la revista emprendía un «ajuste normativo que la homologue con las mejores de su área. El objetivo es facilitar su introducción en las bases de datos más importantes de humanidades y ciencias sociales». «El progreso de la sociedad de la información y la globalización del conocimiento que trae consigo - se añadía - nos demandaban este esfuerzo» ${ }^{12}$. Además de adoptar el concepto de volumen anual, que había llevado a seguir una paginación correlativa en los números del año, la revista comenzó a publicar resúmenes de los artículos, en inglés y en castellano, así como una breve nota curricular de cada uno de los autores. Por otro lado, inaugurando «una nueva etapa de relación con los autores y los lectores», se recogieron también las normas que debían seguir los artículos para su publicación. Finalmente, «la normalización de la revista es también una promesa de mayor regularidad en la aparición de los distintos números».

Por lo demás, la revista no conoció cambios significativos en los años siguientes. Continuó publicando dosieres, dirigidos casi siempre por miembros del Consejo de Redacción, profesores de la Universidad del País Vasco: sus contenidos resultaron representativos de un panorama historiográfico como el español cada vez más diverso en lo temático, teórico y metodológico. En el año 2001 aparecieron los números 22 y 23, dedicados respectivamente a «Cine e historia», bajo la coordinación de Santiago de Pablo, y «Nuevos estudios sobre las élites», dirigido por Luis Castells, quienes firmaron a su vez las introducciones de dichos números. En el 2002, el turno le correspondió a José María Beascoechea Gangoiti y Pedro A. Novo López (número 24, dedicado a «Historia Urbana») y a Carlos Larrinaga (número 25, «Turismo y nueva sociedad»). En 2003, el número 26 fue dirigido por José Antonio Pérez («Desarrollismo, dictadura

12 Esta nota titulada «A los lectores» aparecía firmada por «Historia Contemporánea», pero no resulta difícil percibir en ella la inspiración del director de la revista, Manuel González Portilla. 
y cambios sociales») y el 27 por Javier Fernández Sebastián («Conceptos políticos. Opinión pública. Intelectual»), quien también coordinó -en este caso junto con Iñaki Iriarte López - el número 28, el primero del año 2004, dedicado a «Pueblo, ciudadanía y otros conceptos políticos». La revista sirvió así, de nuevo, para la publicación de las actas de un congreso, en este caso de una selección de los mejores trabajos presentados al V Congreso Internacional de Historia de los Conceptos, celebrado en Vitoria y en Bilbao en 2003.

A partir del número 29, también correspondiente al año 2004, la revista contó más a menudo con coordinadores de dosieres procedentes de otras universidades. Dicho número 29, sobre «La "cuestión social" en la España liberal» fue dirigido por Manuel Suárez Cortina, de la Universidad de Cantabria. Los correspondientes al año 2005 (el número 30, sobre «Franquismo, política exterior y memoria histórica» y el número 31, «Historia de la familia, propuestas transdisciplinares») fueron coordinados, respectivamente, por Juan Carlos Pereira (profesor de la Universidad Complutense) y Ricardo Miralles y por Isabel Moll (de la Universidad de las Islas Baleares). En 2006, el informe sobre «El Mediterráneo: un espacio de conflictos y de encuentros» (número 32) estuvo al cuidado de Antoni Segura (Universidad de Barcelona) y el titulado «El primer constitucionalismo hispanoamericano» (número 33) de José María Portillo. Los números correspondientes al año 2007 -el 34, «La política exterior de España, 1834-1931» y el 35, «La Guerra Civil en el País Vasco: un balance histórico»- fueron coordinados, respectivamente, por Juan Carlos Pereira (Universidad Complutense) y Juan Bautista Vilar (Universidad de Murcia) y por José Luis de la Granja, Santiago de Pablo y Ricardo Miralles.

El año 2008, a partir del número 36, cuando se cumplían 20 años desde la fundación, asiste a una nueva renovación de la revista, en la línea de normalización internacional y de búsqueda de un mayor impacto editorial que se había iniciado años atrás. Manuel González Portilla siguió encargándose de la dirección, mientras que como subdirectores actuaron Luis Castells y Manuel Montero y como secretarios José María Beascoechea y Rafael Ruzafa. El Consejo de Redacción se abrió por primera vez a miembros de otras universidades españolas (Cantabria, Pública de Navarra, Salamanca, Sevilla y Valladolid) y el Consejo Asesor, que había seguido constituido por las mismas personas desde los primeros números de la revista, también se renovó, rejuveneció y amplió profundamente, con historiadores de nuevos países hasta entonces no represen- 
tados (Argentina, Brasil, Italia, México y Portugal) y con un incremento significativo de la presencia de historiadoras. Además, la estructura de cada número resultó simplificada, con tres únicas secciones, dedicadas al dosier, a la miscelánea y a las reseñas. Las presentaciones de cada número dejaron también de referirse al conjunto del volumen y se circunscribieron al apartado monográfico. Pero, según se advertía en la presentación del número 36, el cambio principal residió en que a partir de entonces se utilizó sistemáticamente «la evaluación por parte de informantes externos mediante el sistema de doble ciego, habitual entre las principales revistas científicas» y en que se abría la posibilidad de que, en el futuro, pudieran llegar a publicarse números exclusivamente misceláneos.

Los temas que abordaron los dosieres publicados en 2008 (números 36 y 37) fueron «Entre la historia política y la historia sociocultural» (a él se incorporaron nueve artículos muy diversos, sin que se hiciera constar la existencia de ningún coordinador) e «Investigaciones recientes sobre el republicanismo en España», coordinado por Mikel Urquijo. En 2009 se editaron, por última vez, los resultados de un congreso, en este caso los del coloquio internacional «Reflexiones en torno a la Guerra Civil Española: Historiografías / Paradigmas / Revisionismos», celebrado en Tel Aviv el año anterior, con una presentación firmada por Silvina Schammah Gesser (Universidad Hebrea de Jerusalén), Raanan Rein (Universidad de Tel Aviv) y Javier Ugarte (número 38) y un dosier presentado por Pedro A. Novo López titulado «Nuevas perspectivas sobre la ciudad contemporánea» (39); en este número 39 Historia Contemporánea publicó por primera vez la relación de informantes que habían intervenido en la evaluación de los artículos publicados en 2008 y $2009^{13}$.

En los tres años siguientes la revista no conoció ningún cambio significativo. En 2010 los dosieres versaron «Acerca del País Vasco Contemporáneo» (número 40, que incluyó cinco artículos de historiadores noveles, sin coordinador) y sobre «Gibraltar. Identidad en tiempos de paz y de conflictos» (número 41, coordinado por Rafael Sánchez Mantero, de la Universidad de Sevilla). En 2011 los temas fueron «Regiones económicas en la España Contemporánea» (número 42, dirección de Carlos Larrinaga) y «Doctrina y práctica del liberalismo: la cultura liberal» (número

13 Desde entonces la publicación de la nómina de evaluadores se realizaría con plena normalidad. En el número 43, de 2011, aparecieron los que habían participado en 2010 y 2011. En el número 47, de 2013, los correspondientes a 2012 y 2013. En el 51, de 2015, los de 2014 y 2015. En el 55, de 2017, los de 2016 y 2017. 
43, con presentación de Antonio Morales Moya y Margarita Márquez Padorno, de la Fundación Ortega-Marañón). Y en 2012, «Mujeres, trabajo y recursos para el bienestar» (número 44, dirigido por Pilar Pérez-Fuentes) y «Nacionalismo y homogeneización» (número 45, bajo la coordinación de Daniele Conversi). En este número 45, avanzando en el camino repetidamente anunciado por los responsables de la revista, se incorpora un listado, ya muy voluminoso, de las bases de citas, sumarios y repertorios y bases de datos valorativas donde Historia Contemporánea se encontraba ya recogida e indexada ${ }^{14}$.

En 2013, transcurridos veinticinco años desde la creación de la revista, esta conoció nuevos cambios, los últimos de los impulsados bajo la dirección de Manuel González Portilla, que a su vez fueron consecuencia de la progresiva maduración de una publicación que no solo había mantenido una elevada calidad en sus contenidos, sino que también había conseguido cumplir con los requisitos formales necesarios para incrementar su impacto internacional. Una «Nota de la dirección», publicada en el número 45, daba cuenta de dichos cambios. En primer lugar, una actualización de su diseño exterior, emparentado con el que había mantenido desde su fundación, pero basado ahora en una nueva combinación de colores, con base en el azul y el negro, que, a diferencia de lo que había sucedido hasta entonces, no variaría en cada número. En segundo lugar, un cambio en su organización, que pasó a definirse como un «Consejo editorial», con director, vicedirectores, editores (y ya no secretarios), vocales (en lugar de Consejo de Redacción) y Consejo Asesor. Y finalmente, en cuanto a los contenidos, a partir de esta fecha la revista se abrió a la incorporación de textos en inglés y, para dar salida a la afluencia creciente de originales informados muy favorablemente por los evaluadores, llevó a la práctica la posibilidad apuntada números atrás de publicar números exclusivamente misceláneos: este número 46 fue el primero de Historia Contemporánea que tuvo este carácter, incluyendo once trabajos que, como se indicaba en la presentación, ofrecían «una panorámica abierta de la historiografía que se dirige a nuestra revista desde bien distintas regiones del mundo», pues no en vano dos aportaciones procedían de universidades argentinas y una de una universidad italiana.

14 A partir del número 49 (2014), la revista puedo hacer constar, además, que estaba reconocida con el sello de calidad de la Fundación para la Ciencia y la Tecnología (FECYT). 
A partir de entonces, los números misceláneos convivieron ya con aquellos que incluían un dosier. Estos últimos - como los publicados en años anteriores - resultaron significativos de los notables cambios de tendencia experimentados en los últimos treinta años por la historiografía española: resumiendo mucho, como ha indicado recientemente Javier Rodrigo, se había pasado «de la clase a la cultura y de la cultura a la identidad», hasta acceder a una situación en la que «la tendencia actual de la historiografía es que no existe una tendencia en sí misma» ${ }^{15}$. Los últimos dosieres estuvieron dedicados a «Navarra en sus símbolos, símbolos de Navarra» (número 47, de 2013, coordinado por Jesús Casquete), «Comensalidad y alimentación en la época contemporánea» (número 48, de 2014, dirigido por Jorge Uría y Luis Benito García Álvarez, de la Universidad de Oviedo), «Familias y mujeres en la sostenibilidad de elites y pobres (siglos XVIII y XIX)» (número 49, de 2014, coordinado por Pedro Carasa, de la Universidad de Valladolid), «Modernidad y catolicismo. Nuevas perspectivas sobre una relación compleja» (número 51, de 2015, presentado por Joseba Louzao, de la Universidad de Alcalá de Henares) y «Jóvenes comprometidas en el antifranquismo y la democracia» (número 54, de 2017, bajo la dirección de Mónica Moreno Seco y Adriana Cases-Sola, de la Universidad de Alicante). Por su parte, los números 50 (2015), 52 y 53 (2016) y 55 (2017) tuvieron carácter misceláneo.

Con el número 54 se produjo también una renovación de la organización interna de la revista. A un lado quedaron el director, Manuel González Portilla, y los editores, José María Beascoechea y Eduardo J. Alonso, quien había sustituido en esa función a Rafael Ruzafa a partir del número 51. A otro, el Consejo Editorial, cuya composición se renovó con la salida de algunos miembros y la llegada de otros, como las profesoras Ángela Lucia de Araújo Ferreira (Universidade Federal do Rio Grande do Norte de Brasil) y Charlotte Vorms (Université Paris 1 Panthéon-Sorbonne). Y finalmente, el Consejo Asesor, cuya renovación quedó pendiente para años posteriores.

\section{Epílogo: Historia Contemporánea en 2018}

El año 2018, en el que se cumplían tres décadas desde la fundación de Historia Contemporánea, arrancó con nuevos cambios, que vinieron a con-

15 Rodrigo, 2017, p. 34. 
solidar la transformación iniciada años atrás. Por un lado, la fortaleza y regularidad de la publicación permitió desde el número 56 modificar la periodicidad semestral y hacerla cuatrimestral, pasando a editarse tres números al año, en febrero, junio y octubre. Tras su jubilación, Manuel González Portilla abandonó la dirección y esta pasó a ocuparla José María Beascoechea, con Eduardo J. Alonso Olea como editor, lo que resultaba representativo del cambio generacional que se había producido y que la revista encarnaba con gran plasticidad. Finalmente, la posibilidad anunciada años atrás de que Historia Contemporánea publicase textos en inglés se verificó en este número, con la edición del trabajo «The Best Sheepherder. The Racial Stereotype of Basque Immigrants in the American West between the End of Nineteenth and the Beginning of the Twentieth Centuries», firmado por Iker Saitua. Este número 56 volvió a tener carácter misceláneo, mientras que el segundo del año 2018, el 57, incluyó un dossier sobre «Música popular, identidad y política», coordinado por Paul Ward (Edge Hill University, del Reino Unido) y Ander Delgado, con un nuevo artículo en lengua inglesa.

Al conmemorar sus primeros treinta años de vida, la revista Historia Contemporánea había recorrido un largo camino. Desde los tiempos de su fundación por Manuel Tuñón de Lara, había sido - y probablemente nunca dejó de serlo - una revista de referencia para el contemporaneísmo español. Pero andando el tiempo no lo fue solo por la calidad de sus contenidos y su apertura a los sectores más destacados de la historiografía española, sino también por su capacidad de adaptación a la profunda transformación que el mundo de las publicaciones científicas experimentó durante estos años, un proceso que Historia Contemporánea abrazó en fechas bastante tempranas y que en 2018 había ya culminado, con magníficos resultados en su prestigio académico e impacto editorial. Plenamente asumidas por la revista las nuevas lógicas de la investigación global, solo quedaba por determinar hasta qué punto sería capaz de incrementar en el futuro su presencia internacional, en un contexto en el que la historiografía española seguía mostrando carencias importantes en su nivel de internacionalización.

\section{Bibliografía}

Aróstegui, Julio: «Manuel Tuñón de Lara y la construcción de una ciencia historiográfica», en GRANJA, José Luis de la y REIG TAPIA, Alberto (eds.), $M a$ nuel Tuñón de Lara: el compromiso con la historia, su vida y su obra, Bilbao, Universidad del País Vasco, 1993, pp. 143-196. 
Esteban De Vega, Mariano (ed.), 25 años de historia. La revista Studia Historica en la historiografia española, Salamanca, Ediciones Universidad de Salamanca, 2009.

Granja, José Luis de la y Reig TAPIA, Alberto (eds.), Manuel Tuñón de Lara: el compromiso con la historia, su vida y su obra, Bilbao, Universidad del País Vasco, 1993.

Granja, José Luis de la, Reig TaPia, Alberto y Miralles, Ricardo (eds.), Tuñón de Lara y la historiografía española, Madrid, Siglo XXI, 1999.

Marín GeLABERT, Miquel Àngel, «Ayer. Luces y sombras del contemporaneísmo español en la última década», Ayer, 41, 2001, pp. 213-255.

Marín Gelabert, Miquel Àngel, «Orígenes y primeros años de la Asociación de Historia Contemporánea», Ayer, 92, 2013 (4), pp. 239-250.

MiRALles, Ricardo, «Una empresa historiográfica con autor. Manuel Tuñón de Lara y la revista Historia Contemporánea», Historia Contemporánea, 16, 1997, pp. 13-22.

PÉrez Garzón, Juan Sisinio, «La historiografía en España. Quiebras y retos ante el siglo XXI». En CAmpo, Salustiano del y Tezanos, José Félix (dirs.): España Siglo XXI, vol. 5: Literatura y Bellas Artes, Madrid, Biblioteca Nueva, 2009, pp. 223-260.

RODRIGO, Javier, «¿Quiénes somos? ¿De dónde venimos? ¿Adónde vamos? Algunas reflexiones sobre historiografía y contemporaneísmo en la España del siglo XXI», Studia Historica. Historia Contemporánea, 35, 2017, pp. 23-42.

\section{Datos del autor}

Mariano Esteban de Vega (maes@usal.es) es catedrático de Historia Contemporánea de la Universidad de Salamanca, institución de la que fue director de su Servicio de Publicaciones (entre 2003 y 2007) y vicerrector (entre 2009 y 2017).

Es autor de más de un centenar de publicaciones, entre libros, capítulos de libros, ediciones y artículos en revistas académicas. Sus principales campos de investigación han sido los orígenes de la política social en España y el estudio de los nacionalismos en la España Contemporánea

En la actualidad coordina un proyecto de investigación nacional dedicado a "Nación y nacionalismo en España y América Latina», dentro del Instituto de Iberoamérica de la Universidad de Salamanca. 